\title{
APRENDIZAGEM BASEADA EM PROBLEMAS: UMA EXPERIÊNCIA NO ENSINO DE PATOLOGIA E REABILITAÇÃO DE ESTRUTURAS
}

\author{
MEDEIROS, MARCELO H. F. \\ Professor Dr., Engenheiro Civil \\ Universidade Federal do Paraná \\ Paraná; Brasil \\ medeiros.ufpr@gmail.com
}

\author{
AMARAL, MARCELLA \\ Mestranda, Engenheira Civil \\ Universidade Federal do Paraná \\ Paraná; Brasil \\ marcellamarp@gmail.com
}

\author{
LANGARO, ELOISE A. \\ Doutoranda, Engenheira Civil \\ Universidade Federal do Paraná \\ Paraná; Brasil \\ elolangaro@hotmail.com \\ CALVO, ANALIET \\ Doutoranda, Engenheira Civil \\ Universidade Federal do Paraná \\ Paraná; Brasil \\ analietcv@yahoo.es
}

\section{RESUMO}

A aprendizagem baseada em problemas, em inglês Problem Based Learning (PBL), tem sido muito discutida no Brasil como um meio importante de tornar o ensino mais interessante e interativo para o público alvo. Acompanhando esta tendência, em 2013 esta técnica foi implementada na disciplina TC 083 - Patologia e recuperação de estruturas do curso de Engenharia Civil da UFPR. O presente trabalho tem como foco descrever o formato de aplicação adotado e suas vantagens, através da avaliação qualitativa realizada pelos alunos. Para tal, 5 situações problemas foram idealizadas através da experiência de trabalho na área de patologia das construções do professor da disciplina, Dr. Marcelo Medeiros. Durante o primeiro terço do período do curso, a classe foi dividida em grupos de 4 a 5 alunos e cada situação problema designada a um grupo. Ao longo das aulas, o trabalho foi desenvolvido e evoluiu com a supervisão do professor em conjunto com alunos de pós-graduação em estágio de docência. A participação dos pós-graduandos é fundamental para o bom andamento dos trabalhos, visto que a aprendizagem baseada em problemas exige acompanhamento contínuo para que seja efetivo. Ao final, os alunos responderam um questionário com o intuito de avaliar a experiência vivenciada com esta técnica de ensino. Após os anos de experiência, verificou-se que os alunos apresentaram maior grau de satisfação e participação ao aplicar esta técnica, visto que eles sentem como se estivessem no seu exercício profissional como engenheiros. Além disso, os alunos chegam à conclusão que a base teórica adquirida ao longo da graduação é efetivamente importante, pois eles a usam para desenvolver seus trabalhos durante a disciplina. Palavras-chave: Aprendizagem Baseada em Problemas, patologia das construções, ensino sobre patologia das construções.

\section{ABSTRACT}

The Problem Based Learning (PBL) has been widely discussed in Brazil as an important mean of making the learning more interesting and interactive for the students. In 2013, this technique was implemented in the subject TC $083-$ Pathology and Rehabilitation of structures from Civil Engineering graduation course of Universidade Federal do Paraná. Therefore, this work aims to describe the format of learning used and its advantages through a qualitative evaluation done with the students. During the period of classes, five problem situations were idealized using the expertise of the professor Dr. Marcelo Medeiros in the building's pathology area, the students were divided into groups of four to five and each situation was presented to them in the first third of the course period. Throughout the classes, the work evolves under the supervision of the professor and one or two postgraduate students in teaching internship (masters or doctoral). The participation of postgraduate students is essential to ensure the great progress of the work, since the Problem Based Learning requires continuous monitoring to be effective. At the end of the course, the students answered a questionnaire in order to evaluate their experience with this teaching technique. After the years of experience, it is possible to note a higher degree of satisfaction and participation from the students when the PBL technique was applied during the course. Through the qualitative evaluation, the PBL allows the student to feel as if they were in their professional practice as engineers. Moreover, they come to the conclusion that the theory that they have learned are indeed important, since they can apply their knowledge to develop their work during the course.

Keywords: Problem Based Learning (PBL), building's pathology, building's pathology teaching. 


\section{INTRODUÇÃ̃O}

A utilização de novas tecnologias e estratégias pedagógicas podem valorizar a formação de profissionais e revolucionar vários segmentos de ensino. A formação de engenheiros, por exemplo, precisa ser cada vez mais debatida e estudada em nível nacional, visto que ainda existe pouca atuação de organismos ligados a ensino em engenharia. No Brasil, um exemplo positivo neste campo é a Associação Brasileira de Educação em Engenharia (ABENGE), cuja missão é "produzir mudanças necessárias para melhoria da qualidade do ensino de graduação e pós graduação em engenharia e tecnologia no Brasil, contribuindo decisidamente para a formação de profissionais cada vez mais qualificados e capacitados que levem o desenvolvimento e tecnologia a todos os pontos do país pelos benefícios que a engenharia pode proporcionar a toda população."

A ABENGE promove anualmente, desde 1973, um evento intinerante chamado COBENGE (Congresso Brasileiro de Educação em Engenharia), que é o mais importante fórum de discussão sobre a formação e o exercício profissional em Engenharia no Brasil. Este evento vem, nos últimos anos, debatendo filosofias e paradigmas do processo de ensino e aprendizagem de Engenharia e Tecnologia.

Neste contexto, de acordo com Suguinoshita et al. (2016), baseados em experiências realizadas no Canadá e na Holanda, várias escolas do Brasil vêm buscando adotar a metodologia do PBL (Problem Based Learning - em português, Aprendizagem Baseada em Problemas) em seus currículos. O método se trata de uma ferramenta inovadora utilizada na educação pelo qual os alunos investigam e solucionam um problema proposto trabalhando em grupo, tendo o senso crítico e trabalho em equipe desenvolvidos, além de instigação da aprendizagem ativa.

Camargo Ribeiro (2008) defende que uma das vantagens mais citadas do PBL é a capacidade de tornar a aprendizagem mais dinâmica e prazerosa, compartilhada entre discentes e docentes. Ela confere aos alunos uma maior motivação para o trabalho desde os primeiros anos de formação e fomenta um ambiente de aprendizado onde o companheirismo é estimulado, uma vez que os alunos estão divididos em grupos de trabalho. Os estudantes aprendem a respeitar opiniões diversas e a construir consensos. Gerez et al. (2019) ressaltam que a utilização de metodologia ativa desenvolve, também, habilidades não técnicas, mas que são essenciais a um profissional de engenharia que na maioria das vezes está limitado ao conhecimento técnico apenas.

Tendo como motivação essa necessidade de mudanças no modelo educacional dos cursos de Engenharia Civil, este trabalho registra a experiência da aprendizagem baseada em problemas implementada na disciplina TC 083 (Patologia e recuperação de estruturas) no curso de graduação em Engenharia civil da Universidade Federal do Paraná, sendo descritas as 5 situações problemas que foram idealizadas para a disciplina, assim como a percepção dos alunos participantes deste processo.

\section{METODOLOGIA}

\subsection{Aplicação da técnica PBL}

A técnica de aprendizagem baseada em problemas foi aplicada no trabalho final da disciplina TC083 - Patologia e reabilitação de estruturas do curso de graduação em Engenharia civil da Universidade Federal do Paraná. A disciplina é optativa para os estudantes e possui uma carga horária de 60 horas-aula, sendo disponibilizadas 45 vagas para os alunos regularmente matriculados no curso de graduação em questão.

Durante o período de aulas, os alunos da disciplina foram divididos em grupos com no máximo 5 integrantes para a realização de um trabalho com o intuito de aproximar as problemáticas reais ao conteúdo teórico explanado pelo professor. O plano de ensino da metodologia utilizada dividiu a proposta em 7 etapas, como mostra a Figura 1, sendo possível acompanhar o roteiro lógico descrito por Ribeiro (2008) para PBL, que consiste em: (a) definição do problema de estudo com uma questão motivadora; (b) desenvolvimento do pensamento crítico dos alunos acerca do problema proposto; (c) escolha de uma solução a partir de diversas referências pesquisadas e (d) divulgação do projeto possibilitanto uma forma de feedback. 




Figura 1: Etapas de desenvolvimento do trabalho aplicado aos estudantes.

Em um primeiro momento, cada grupo recebeu a descrição de uma edificação contendo um problema construtivo que deveria ser identificado, sendo necessário elaborar uma proposta comercial com os serviços necessários para identificar a anomalia presente na situação. Cabe ressaltar que o trabalho foi proposto de forma que os alunos atuassem como uma empresa de engenharia diagnóstica, desenvolvendo todas as etapas comerciais necessárias no mercado profissional para a análise de uma edificação. Para dar uma perspectiva das situações enfrentadas pelos alunos, um resumo das mesmas será descrito no item 2.2 .

Posteriormente, o professor, com o auxílio dos alunos em estágio de docência, desempenhou o papel dos clientes e avaliou as propostas desenvolvidas, enviando uma contraproposta que levantava possíveis hipóteses, indagava a escolha dos procedimentos e sugeria possíveis alterações. Após os devidos ajustes na proposta comercial, realizou-se a parte de "execução do serviço", em que os alunos receberam um histórico de dados respectivos aos ensaios e procedimentos definidos por eles como essenciais para a composição do laudo final. Os estudantes realizaram a análise dos dados, levantaram as possíveis causas e indicaram as possíveis medidas para reabilitação das edificações em estudo, sendo apresentado um laudo para o cliente contendo todas as etapas de análise e as informações necessárias em relação ao estudo diagnóstico realizado.

Levando em consideração que cada grupo recebeu uma situação problema com diferentes tipos de edificações e situações, ao final do estudo os estudantes realizaram uma apresentação oral descrevendo todo o processo realizado, bem como a proposta de reabilitação sugerida por eles. Além da disseminação do conhecimento agregado durante a atividade, este momento de exposição permitiu que houvesse uma ampliação na visão dos estudantes, visto que foi possível uma troca de opiniões entre futuros profissionais acerca das ações sugeridas por cada grupo.

\subsection{Situações problema}

A situação problema 1 tratou-se de uma edificação construída na orla marítima de Matinhos (Paraná), a qual apresentou problema na concretagem das vigas e lajes do segundo pavimento. Na primeira etapa, foi solicitada uma averiguação da resistência à compressão dos corpos de prova retirados de cada caminhão betoneira. Numa segunda etapa, uma inspeção preliminar foi realizada e nesta observada diversas fissuras nas vigas do segundo pavimento além de pilares com armadura exposta contendo indícios de corrosão. A partir desta inspeção, foi solicitada a elaboração da proposta comercial. Essas duas etapas foram realizadas com todas as 5 situações apresentadas aos alunos com o intuito de simular o primeiro contato com o cliente além de uma visita de inspeção na obra.

Na situação problema 2, da mesma forma que na situação problema 1 , foi verificado problema na concretagem das vigas e lajes do segundo pavimento numa primeira etapa. Além disso, o cliente solicitou uma inspeção após uma estação de chuvas devido a uma infiltração nas lajes do pavimento superior. Esta edificação estava localizada na cidade de Cascavel, Paraná.

A situação problema 3 contou com uma edificação construída em 2005, na cidade de Curitiba (Paraná) e paralisada posteriormente ao encerramento dos trabalhos pela construtora. Na retomada da obra uma inspeção preliminar foi feita e nesta foi averiguado diversos problemas, entre eles elevada fluência em duas lajes com armadura aparente na parte inferior, além de diversos pilares com armadura exposta apresentando corrosão.

Da mesma forma que na situação problema 3, a situação problema 4 contou com a retomada da construção de uma edificação que havia sido paralisada no ano de 1999. Essa edificação foi construída na cidade de Balneário Camboriú, 
Paraná, e após a inspeção preliminar foi verificado que haviam várias fissuras nas vigas e má adensamento do concreto em diversos pontos da estrutura. O cliente solicitou uma proposta que visasse informar as medidas necessárias e imprescindíveis para recuperar a edificação e proceder com a execução da mesma.

A situação problema 5 apresentou uma ponte construída pelo DNIT no ano de 2010. Esse caso objetivava traçar um plano de manutenção preventiva para esta ponte a fim de averiguar o estado atual da mesma, condições de exposição e previsão da vida útil baseada no estado atual e microclima existente na região. Uma inspeção visual foi realizada e nesta constatado pontos de infiltração e diversas fissuras nos elementos construtivos. A partir das informações prestadas foi solicitada a proposta comercial.

\subsection{Percepção dos estudantes quanto a utilização da PBL}

Ao final da disciplina TC 083: Patologia e recuperação de estruturas foi enviado um questionário aos estudantes, relacionado ao desenvolvimento dos projetos utilizando a Aprendizagem Baseada em Problemas. O questionário foi elaborado com 13 questões, divididas em grupos de acordo com os aspectos abordados, como mostra o Quadro 1. As perguntas do item (a) tiveram como objetivo abordar a qualidade da disciplina pelo ponto de vista de estudante, podendo auxiliar no melhoramento dos objetivos e temas abordados nas aulas teóricas. O item (b) consistiu em uma avaliação da técnica de aprendizado baseada na resolução de problemas aplicada no trabalho final da disciplina, buscando entender o nível de satisfação dos estudantes com o método. Por fim, o item (c) engloba questões relacionadas ao armazenamento e aplicação do conhecimento adquirido ao longo do período de estudos, com o intuito de avaliar a relevância da disciplina para a formação do profissional de Engenharia Civil.

As perguntas do questionário se dividem em dois grandes grupo, sendo algumas avaliadas de maneira qualitativa pelos estudantes, através de uma escala gráfica com pontuação de 1 a 5 (1-Muito baixo; 2-Baixo; 3-Razoável; 4-Alto; 5Muito alto) e outras de forma dicotômica, em que o estudante poderia escolher apenas uma opção de resposta (Sim/Não/Talvez). Cabe ressaltar que o questionário não foi um teste avaliativo e, por isso, foi mantido o anonimato do estudante ao respondê-lo.

Quadro 1 - Questionário desenvolvido para avaliar a utilização da técnica de aprendizado baseada em problemas.

\begin{tabular}{|c|c|}
\hline Aspectos & Questões \\
\hline $\begin{array}{c}\text { (a) } \\
\text { Qualidade da disciplina }\end{array}$ & $\begin{array}{l}\text { 1. A disciplina foi apresentada, com objetivos claros? } \\
\text { 2. O quanto a disciplina foi relevante para a sua formação?* } \\
\text { 3. A distribuição de conteúdo ao longo do curso foi adequada? } \\
\text { 4. Em uma escala de } 1 \text { a } 5 \text {, como você classifica o grau de dificuldade da disciplina?* }\end{array}$ \\
\hline $\begin{array}{l}\text { (b) } \\
\text { Técnica de aprendizado } \\
\text { baseado na resolução de } \\
\text { problemas }\end{array}$ & $\begin{array}{l}\text { 5. Como você classifica a técnica de aprendizado baseada na resolução de problemas } \\
\text { hipotéticos?* } \\
\text { 6. A forma como foi aplicada a técnica, em diferentes etapas, foi representativa do ponto de } \\
\text { vista de um problema real? } \\
\text { 7. Você conhecia a técnica de aprendizado baseado em problemas ou do inglês PBL } \\
\text { (Problem Based Learning)? } \\
\text { 8. Outras disciplinas cursadas no curso de Engenharia Civil fazem uso da técnica de } \\
\text { aprendizado baseado em problemas ou do inglês PBL (Problem Based Learning)? Se sim, } \\
\text { quais? } \\
\text { 9. A técnica de aprendizado baseado em problemas ajudou a aproximar o entendimento da } \\
\text { atuação do engenheiro na área de Patologia das Construções? }\end{array}$ \\
\hline
\end{tabular}

(c)

Armazenamento e aplicação do conhecimento
10. Como você classifica o seu grau de entendimento nesta disciplina?*

11. Como você classifica o seu grau de envolvimento e esforço nesta disciplina?*

12. Você adquiriu conhecimentos novos com esta disciplina?

13. Após cursar a disciplina, seu interesse pelo assunto aumentou?

*Pergunta com escala gráfica qualitativa (1-Muito baixo; 2-Baixo; 3-Razoável; 4-Alto; 5-Muito alto) 


\section{RESULTADOS E DISCUSSÕES}

A amostragem realizada, a partir da aplicação do questionário, englobou duas turmas que cursaram a disciplina TC 083: Patologia e recuperação de estruturas nos anos de 2018 e 2019. Os três principais aspectos abordados no questionário visaram entender, de um modo geral, a visão do aluno sobre a disciplina, o quanto a técnica PBL foi interessante e pertinente no desenvolvimento da disciplina e o quanto a aplicação dessa técnica aproximou o conhecimento gerado em sala de aula de uma situação real.

No primeiro bloco de perguntas, foi possível observar que a maioria dos estudantes tiveram uma boa percepção sobre a disciplina (questão 2), classificando-a como relevante em sua formação acadêmica (68,8\%). Além disso, apenas 18,8\% compreenderam o grau de dificuldade da mesma como elevado (questão 4), sendo que a grande parte a considerou razoável (62,5\%). Quanto aos objetivos (questão 1) e conteúdo da disciplina abordado (questão 2) durante o desenvolvimento da mesma, uma pequena parcela $(6,3 \%)$ de alunos não considerou a distribuição do conteúdo adequado ao longo do curso, e 12,5\% encontrou alguma dificuldade com a clareza dos objetivos propostos. A Figura 2 mostra os resultados da avaliação dos alunos neste primeiro aspecto.
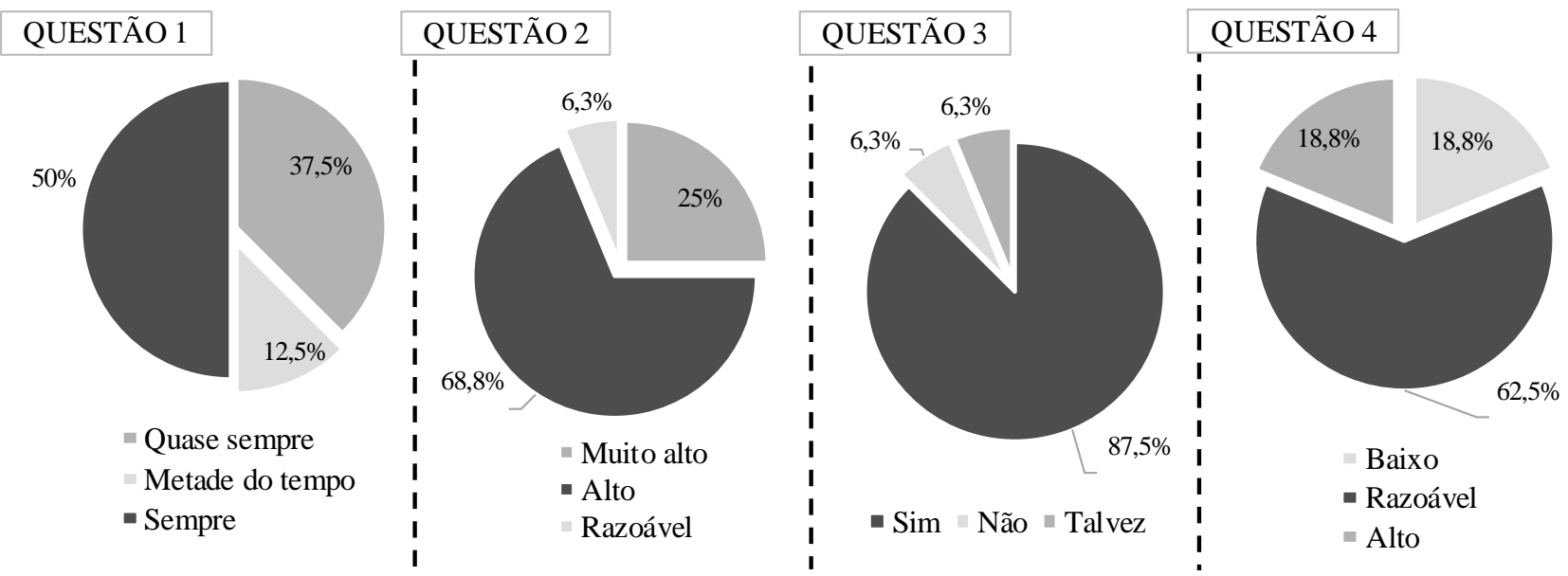

Figura 2: Qualidade da disciplina a partir da percepção dos estudantes.

A importância de avaliar a qualidade da disciplina, pelo ponto de vista do estudante, contribui postivamente para o aprimoramento do conteúdo das aulas, tendo em vista principalmemente os aspectos relacionados as questões 1 e 2 , os quais são primordiais no bom desempenho e comprometimento do aluno.

A técnica de aprendizado baseado na resolução de problemas foi abordada no segundo bloco de perguntas. Os tópicos levantados nesse item visavam entender como o aluno avalia o uso desta técnica mediante o conteúdo abordado. A Figura 3 mostra os resultados obtidos a partir das questões aplicadas.
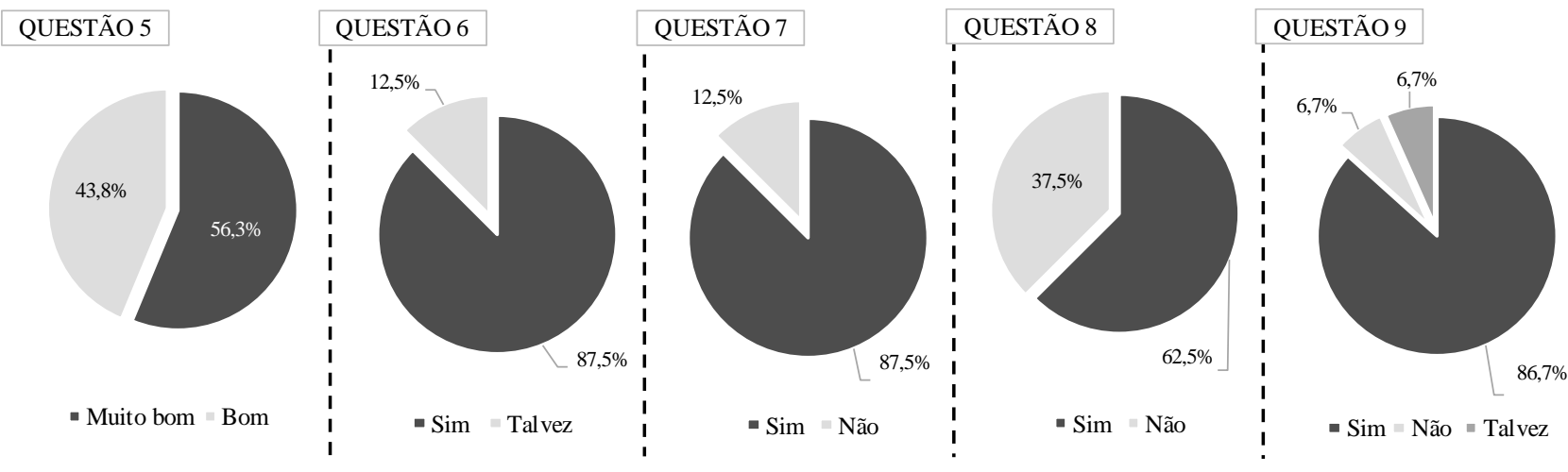

Figura 3: Aplicação da técnica de aprendizado baseado na resolução de problemas com base no ponto de vista dos estudantes. 
Com base nos resultados, foi possível observar a grande aceitabilidade por parte dos alunos pela utilização da técnica PBL, sendo que todos classificaram essa abordagem dentro da disciplina como boa ou muito boa (questão 5), além disso, grande parte dos estudantes já conhecia esse tipo de técnica (questão 7). A questão 9 vem de encontro com a questão 5, pois aborda o uso da situação problema como ferramenta de aproximação da vida profissional do engenheiro. A maioria dos estudantes avaliou de forma positiva o trabalho aplicado usando a metodologia PBL (86,7\%). De acordo com Ribeiro (2016) existe uma construção de conhecimento na aprendizagem utilizando o PBL, ou seja, quando um estudante é exposto a uma situação corriqueira da profissão e precisa desdobrá-la de forma a resolvê-la, ele não está apenas acumulando conhecimento, e sim construindo novas perspectivas e de certa forma experiências que vão auxiliálo positivamente na sua vida profissional posterior.

Dentro da disciplina TC 083: Patologia e recuperação de estruturas, cada grupo de alunos desempenha o papel de empresa diagnóstica e recebe problemas diferentes para resolver. Esses problemas são baseados e desenvolvidos de acordo com as aulas teóricas expostas. A proposta do trabalho em etapas visa aproximar o conteúdo em sala da realidade de um trabalho dessa complexidade. Na questão 6 esse aspecto foi avaliado pelos estudantes e, a maior parte deles $(87,5 \%)$ entendeu que a aplicação do trabalho em etapas foi representativa pelo ponto de vista profissional. Na engenharia, a técnica PBL sofreu alterações na forma de abordagem para se adequar a complexidade dos problemas dentro da área (Ribeiro, 2016), e trabalhar com etapas produz um resultado final melhor, tendo em vista a construção e amadurecimento do conteúdo abordado durante as aulas. Como comenta Furtado et al. (2018), o uso de etapas também tem o objetivo de estimular o foco e motivação entre os alunos e, além disso, os resultados de sua pesquisa mostraram que a atividade em geral contribuiu em habilidades de comunicação e trabalho em equipe.

Visando entender o quanto a técnica PBL era aplicada dentro do curso de Engenharia Civil, a questão 8 foi aplicada no formulário. Além da questão 8, no formulário foi solicitado a indicação das disciplinas que aplicam o método. Apesar de 62,5\% dos estudantes terem apontado como resposta "sim", notou-se que dentro do currículo total de disciplinas, são poucas as que aplicam esse tipo de abordagem. As disciplinas apontadas pelos alunos foram: Pavimentação, Sistema de Transporte e Laboratório de Materiais de Construção Civil. De um total de 64 disciplinas ofertadas pelo curso de Engenharia Civil, apenas 3 foram apontadas, ou seja, apenas 5\% das disciplinas aplicam esse tipo de técnica.

O terceiro bloco de questões visou entender qual era o entendimento do aluno pela disciplna e o quanto cursar a mesma foi interessante pelo ponto de vista de novos conhecimentos e interesse na área específica. A Figura 4 mostra as respostas referentes aos aspectos levantados nesse item. Todas as questões abordadas estão correlacionadas com o uso da técnica PBL.

Notou-se que o grau de entendimento dos conteúdos por parte dos alunos foi relativamente elevado (questão 10). O uso da situação problema, contribuiu positivamente na fixação e aplicação do conteúdo abordado nas aulas teóricas, o que levou os alunos a obterem um grau de satisfação melhor quanto ao aprendizado do conteúdo. A questão 11 mostra o envolvimento dos alunos mediante a disciplina. A observação desse resultado remete diretamente ao sucesso ou não do uso da ferramenta PBL. De acordo com Fernandes et al. (2017), o uso dessa metologia é colaborativa, ou seja, necessita da participação e envolvimento de todos para que seja bem-sucedida. Neste quesito, os autores discutem que, apesar da técnica já ser conhecida na área de engenharia, os alunos ainda estão acostumados ao modelo tradicional passivo de ensino e, portanto, muitos não se comprometem o suficiente para o bom aproveitamento da técnica. Porém, apesar dos resultados não apontarem $100 \%$ de elevado comprometimento com a disciplina, foram satisfatórios visto que o grau de entendimento dos conteúdos (questão 10), por parte dos alunos, foi em geral alto. 


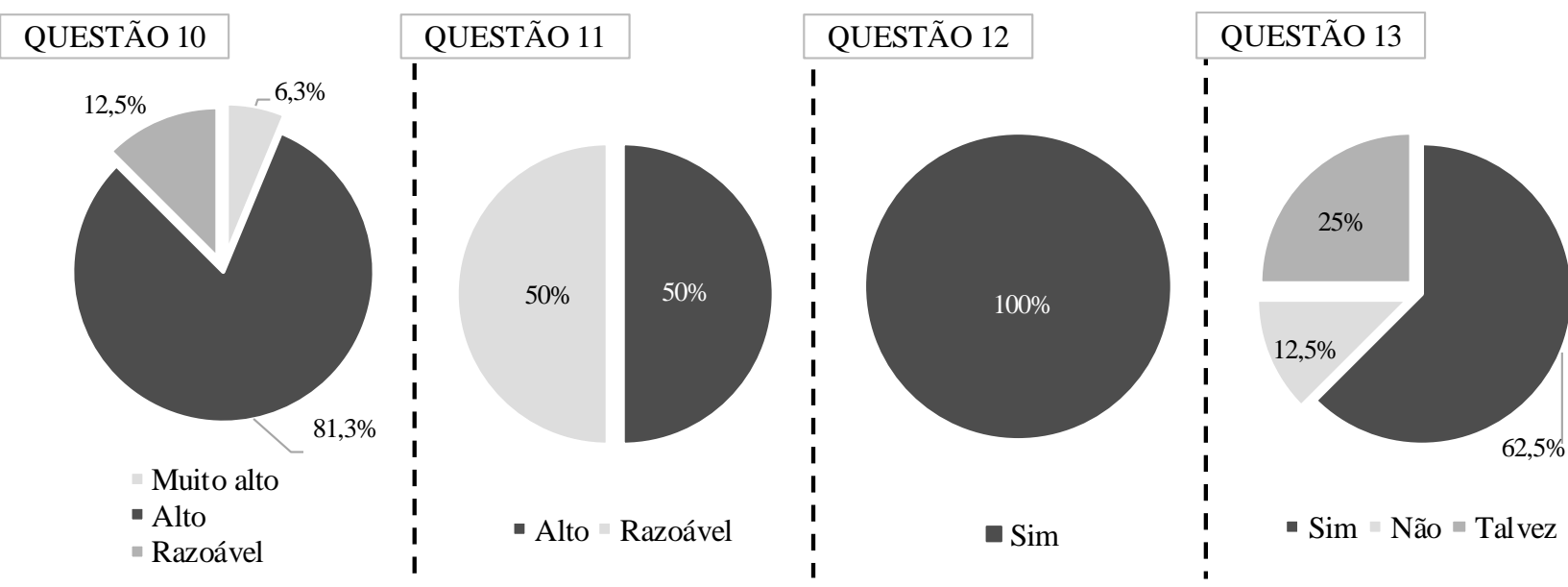

Figura 4: Avaliação quanto ao armazenamento e aplicação do conhecimento.

A questão 12 avaliou o quesito novos conhecimentos adquiridos. Visto que 100\% das respostas foram positivas, a disciplina atingiu, ao que tudo indica, um nível satisfatório de abordagem. Já a questão 13 aborda o interesse pela área após cursar a disciplina. Essa questão vai de encontro com as habilidades do estudante, uma vez que o interesse é maior quando há identificação com o tema da disciplina. Apenas 12,5\% das respostas foram negativas para este quesito.

De forma geral os resultados mostraram que o uso da metodologia PBL, observada nas respostas do formulário, torna a forma de aprender mais dinâmica e motivadora, fazendo com que a busca por conhecimento seja constante e o trabalho em equipe seja desenvolvido.

\section{CONCLUSÕES}

A partir do questionário desenvolvido, com o intuito de obter uma avaliação qualitativa da metodologia do PBL (Problem Based Learning - em português, Aprendizagem Baseada em Problemas), foi possível constatar que desde o ponto de vista dos estudantes a disciplina é relevante para sua formação profissional, considerando o grau de dificuldade razoável a elevado.

Enquanto aos objetivos e conteúdos da disciplina, uma pequena parcela não considerou a distribuição do conteúdo adequado ao longo do curso, e encontraram alguma dificuldade com a clareza dos objetivos propostos. Nesse sentido vale destacar que ainda não existe a obrigatoriedade de especializar-se na área de patologia das construções, para atuar como especialista. Nos cursos de engenharia civil, geralmente a matéria é ofertada como disciplina optativa na graduação. Geralmente, o aluno de graduação recorre a cursos de especialização Latu Senso em patologia das construções como uma maneira de suprir a carência de informações, uma vez que já é um profissional atuante no mercado.

Apesar de tudo, mesmo como optativa, a existência de disciplinas como esta pode ser considerada uma vitória da engenharia civil resultante da militância de vários professores e pesquisadores das universidades nacionais em prol desta vertente de formação necessária atualmente, haja vista a preocupação acerca de construções sustentáveis e mais duráveis.

No ambito geral, a maioria dos estudantes avaliou de forma positiva o trabalho aplicado usando a metodologia PBL, pois as situações problema que eles precisaram desdobrar e resolver, permitiu-lhes não apenas acumular conhecimento, mas construir novas perspetivas sobre como vai ser sua vida profissional no futuro. A metodologia levou os alunos a obterem um grau de satisfação melhor quanto ao aprendizado do conteúdo. Além disso, as atividades contribuiram para aperfeiçoar habilidades de comunicação e trabalho em equipe. Porém, dentro do currículo total de disciplinas do curos de Engenharia Civil da UFPR, são poucas as que aplicam esse tipo de abordagem. 


\section{AGRADECIMENTOS}

Agradecemos a CAPES, Coordenação de Aperfeiçpamento de Pessoal de Nível Superior.

\section{REFERÊNCIAS}

RIBEIRO, Luis Roberto. Aprendizagem baseada em problemas (PBL) na educação em engenharia. Revista de Ensino de Engenharia, v. 27, n. 2, p. 23-32, 2008.

GereZ, J. C.; Rodrigues, G. A. C.; FAGundeS, K. P.; FreitAS, L. G.; OZÓRIO, B. P. M. Análise da aplicação da metodologia PBL (Problem Based Learning) em uma disciplina inical dos cursos de engenharia civil. Cobenge, p. 1-9, 2019.

SUGUINOSHITA, M.C. ; COSTA, R.A. ; BONATTO, I. ; ZAU, S.K.S. ; MIRANDA, D.C. ; CASTELANI, L.V.C. ; ZORZAN, L.G. ; HORTEGA, M.D. ; BUENO, W.M. ; PINTO, M.S.N. ; SONEGO, M.F. ; WOGEL, O.M. ; MEDEIROS, M.H.F. Aplicação de PBL (Project Based Learning) em Disciplinas do Curso de Engenharia Civil da UFPR. In: III Congresso Nacional dos Grupos PET de Engenharia Civil, 2016, Curitiba. Anais do III Congresso Nacional dos Grupos PET de Engenharia Civil. Curitiba: Universidade Federal do Paraná, 2016. v. único.

FURTADO, A. E.; NASCIMENTO, D. F. L.; JESUS SILVA, J. W. Aprendizagem Baseada em Problemas (PBL) aplicada simultaneamente para estudantes de engenharia de $3^{\circ}$ e $7^{\circ}$ períodos como ferramenta motivacional. Revista Práxis, v. 10, n. 19, jun., 2018.

FERNANDES, F.A.S.; SANTOS, E.G.; MORAIS, L.B. Utilização da PBL como metodologias ativas na graduação: área de exata na cidade de Palmas/TO. Revista Educ., Brasília, ano 40, n. 154, p. 192-206, jul./dez. 2017. 